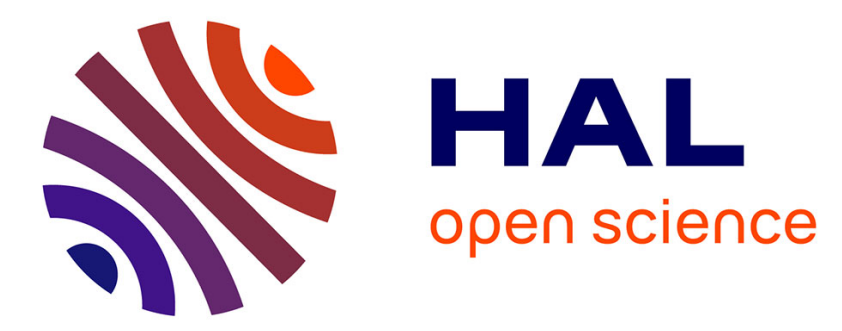

\title{
Robustness Improvement of Compact Predistorters in a CO-OFDM System Using Semiconductor Optical Amplifiers
}

Mohamad Younes, Mihai Telescu, Noël Tanguy, Chérif El Valid Diouf, Pascal Morel, Stéphane Azou

\section{To cite this version:}

Mohamad Younes, Mihai Telescu, Noël Tanguy, Chérif El Valid Diouf, Pascal Morel, et al.. Robustness Improvement of Compact Predistorters in a CO-OFDM System Using Semiconductor Optical Amplifiers. 29th International Conference on Microelectronics (ICM), 2017, Dec 2017, Beirut, Lebanon. 10.1109/ICM.2017.8268828 . hal-01697205

\section{HAL Id: hal-01697205 \\ https://hal.univ-brest.fr/hal-01697205}

Submitted on 31 Jan 2018

HAL is a multi-disciplinary open access archive for the deposit and dissemination of scientific research documents, whether they are published or not. The documents may come from teaching and research institutions in France or abroad, or from public or private research centers.
L'archive ouverte pluridisciplinaire HAL, est destinée au dépôt et à la diffusion de documents scientifiques de niveau recherche, publiés ou non, émanant des établissements d'enseignement et de recherche français ou étrangers, des laboratoires publics ou privés. 


\section{Robustness Improvement of Compact Predistorters in a CO-OFDM System Using Semiconductor Optical Amplifiers}

\author{
M. Younes, M. Telescu, N. Tanguy \\ Université de Bretagne Occidentale \\ CNRS UMR 6285 Lab-STICC \\ Brest, France \\ noel.tanguy@univ-brest.fr
}

\author{
C. Diouf, P. Morel, S. Azou \\ École Nationale d'Ingénieurs de Brest \\ CNRS UMR 6285 Lab-STICC \\ Brest, France \\ azou@enib.fr
}

\begin{abstract}
This paper focuses on digital signal processing techniques compensating the non-linear effects inherent to optical modulation or amplification within a coherent optical orthogonal frequency division multiplexing (CO-OFDM) transmitter featuring a semiconductor optical amplifier (SOA). Two complementary approaches are specifically considered: crest factor reduction and linearization using a digital baseband predistortion. Two possible implementations are presented for the digital predistortion block. One is the basic solution of static compensation; the other consists in a parallel two-box digital polynomial predistorter (PTB) combining, in parallel, a static nonlinear block with a memory polynomial predistorter. Moreover, we show that a linearization of the dynamic behavior of the transmitter, in addition to peak-to-average power ratio (PAPR) reduction, offers better performance. The robustness of the predistorters or of the combined predistorter/PAPR reduction blocks is assessed for various scenarios. Finally, we propose solutions to improve robustness via multipoint identification or switching strategies for predistortion.
\end{abstract}

Keywords-Coherent Optical OFDM; Semiconductor optical amplifier (SOA); Digital Predistortion; Linearization; PAPR reduction; Robustness analysis; Error Vector Magnitude (EVM).

\section{INTRODUCTION}

Orthogonal frequency division multiplexing (OFDM) offers many advantages for future high-speed optical communication networks [1], in particular bandwidth allocation flexibility and efficient compensation of channel imperfections in frequency domain by digital signal processing. Recently, the practical feasibility of amplification of coherent optical OFDM signals by semiconductor optical amplifiers (SOAs) has been demonstrated [2], but the intrinsic nonlinear effects to this type of component, resulting from its fast gain dynamics, can in some cases affect the performance of the system. Linearization is a classic way of reducing the impact for non-linearities in telecommunication systems and digital baseband predistortion is an interesting linearization strategy. It has the ability to meet both performance criteria, i.e. decreasing the error vector magnitude (EVM) and flexibility criteria. Ideally, predistortion is an inverse numerical model of the source of non-linear effects (essentially the SOA in our case). A large diversity of digital predistortion solutions is studied in literature, mainly for the case of radiofrequency systems [3]. The interest for this type of processing in optical systems is much more recent [4] [5]. The Memory Polynomial (MP) model, or its generalized formulation (GMP), is extensively adopted for its good performance. The interest of using a simple look-up-table (LUT) design is mentioned in a few references [3] when the nonlinearity under test can be assumed as memoryless.

In our previous study [6], we compared the basic solution of static nonlinearity (Static predistorter) with an extended solution featuring the addition of a memory polynomial (parallel two-box digital polynomial predistorter - PTB) in order to handle the memory effects of the amplifier. We also analyzed the robustness of the two schemes in the presence of parameter variations in the transmitter. In this paper, we extend this study, in order to improve the robustness, by combining predistortion with a simple PAPR reduction law via hardclipping. We show that this combination offers better performance when several system parameters vary. These parameters are of physical nature, such as the peak-to-peak voltage of the Mach-Zehnder modulator, optical power, bias current for the SOA, and laser wavelength. To the best of the authors' knowledge this sort of analysis has not yet been conducted although it is extremely useful in the perspective of meeting future network demands, involving possibly adaptive transceiver parameters [7].

\section{CO-OFDM SYSTEM MODEL}

The results presented throughout the paper are obtained using Matlab-ADS co-simulation [5], [6]. The CO-OFDM transmitter and receiver (Fig. 1) are modeled in Matlab while the SOA is modeled, using carrier density rate and optical signal field propagation equations, in ADS Ptolemy. On the transmitter side, non-linearities are generated by the optical amplifier (SOA) and to a lesser extent by the IQ modulator. The imperfections of the RF circuits upstream of the modulator are ignored. For the SOA, we use the same model as in [2], optimized to simulate a commercial component (INPHENIXIPSAD1501) which has already yielded an excellent match between simulation and experimental results. The structures of the transmitter and the receiver are conventional, except for the blocks dedicated to linearization (via Static or PTB) and the reduction of PAPR via hard clipping. Subsequently, we focus on the case of a QPSK/OFDM transmission of $20 \mathrm{GHz}$ bandwidth with 512 subcarriers and peak-to-peak voltage equal 
to $8 \mathrm{~V}$; the $200 \mathrm{~mA}$ polarized amplifier provides a gain of 19 $\mathrm{dB}$ at the wavelength of $1540 \mathrm{~nm}$ for a noise factor of $7 \mathrm{~dB}$. The peak-to-average power ratio (PAPR) reduction is a classic approach aimed at limiting the high amplitudes of an OFDM signal. Thus nonlinear distortions, which can result from optical amplification, are reduced. However, the PAPR reduction does not correct the nonlinearities of the transmitter. The joint use of PAPR reduction and linearization via digital predistortion is investigated in this paper, with the view to improve the performances of coherent optical OFDM systems employing a semiconductor optical amplifier.

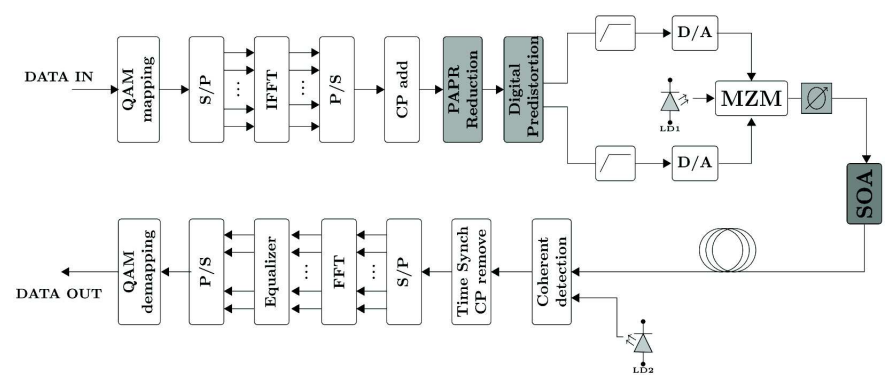

Fig. 1. CO-OFDM structure (see [6] for further details).

\section{PRedistortion Structures}

Predistortion can be used for various non-linear components. The general concept behind predistortion is to precompensate impairments by distorting the signal injected into the amplifier. Two polynomial-based structures will be comparatively used to model the SOA inverse function: the Static predistorter and the PTB predistorter. The PTB is a parallel association of a memoryless structure and an MP block. A short presentation of both concepts follows, summarizing the in-depth study available in [6].

\section{A. Memoryless Polynomial Predistorter (Static)}

The Static predistorter characterized by the input-output relation (1) offers the possibility of adjusting the orders of nonlinearity $N_{1}$, and $N_{2}$ where $|\mathrm{x}[\mathrm{k}]|$ and $\varphi_{x[k]}$ denote input electrical magnitude and phase, respectively:

$$
y[k]=H_{S T}\{x[k]\}=G(|x[k]|) e^{\left(j \varphi_{x[k]}+\Delta_{A M / P M}(|x[k]|)\right.}
$$

$G($.$) and \Delta_{A M / P M}($.$) are two polynomials of |\mathrm{x}[\mathrm{k}]|$ of $N_{1}$ and $N_{2}$ orders. SOA amplitude-amplitude distorsions are compensated by predistortion gain $G($.$) while SOA amplitude-$ phase impairments are compensated by phase shift $\Delta_{A M / P M}$ :

$$
\begin{gathered}
G(|x[k]|)=\sum_{n=0}^{N_{1}} a_{n}|x[k]|^{n} \\
\Delta_{A M / P M}(|x[k]|)=\sum_{n=0}^{N_{2}} b_{n}|x[k]|^{n}
\end{gathered}
$$

Parametric complexity amounts to $N_{1}+N_{2}+2$.

\section{B. Parallel Two-Box Digital Polynomial Predistorter (PTB)}

For a discrete input signal $x[k]$ and the predistorter output $y[k]$, the PTB structure is described by

$$
y[k]=H_{P T B}\{x\}=H_{S T}\{x\}+H_{M P}\{x\}
$$

where $H_{S T}\{x\}$ is given by (1) and

$$
H_{M P}\{x\}=\sum_{l=1}^{N_{3}} \sum_{m=0}^{M-1} d_{l, m} x[k-m]|x[k-m]|^{l-1} ; l \text { odd }
$$

The PTB structure has an additional degree of freedom on the memory depth $M$.

Parametric complexity amounts to $N_{1}+N_{2}+2+\left\lfloor\frac{N_{3}+1}{2}\right\rfloor M$.

\section{Predistorter Identification}

Identification consists in the computation of the considered model coefficients. As predistortion is carried out in electrical domain, identification stimuli are the transmitted electrical signal $x$, just before $\mathrm{E} / \mathrm{O}$ conversion and the received electrical signal $x_{r}$, just after $\mathrm{O} / \mathrm{E}$ conversion. Accordingly a simulation with a $2^{11}$ 4-QAM symbols input sequence is first performed without predistortion in the transmission chain. Then signals $x$ and $x_{r}$ are retrieved from simulation results and used to compute predistorter coefficients using least squares (LS) algorithms. Static and PTB will accordingly be identified by an offline LS algorithm which relies on a QR decomposition for better numerical stability [8].

\section{RoBUSTNESS ANALYSIS}

\section{A. Predistortion Performance}

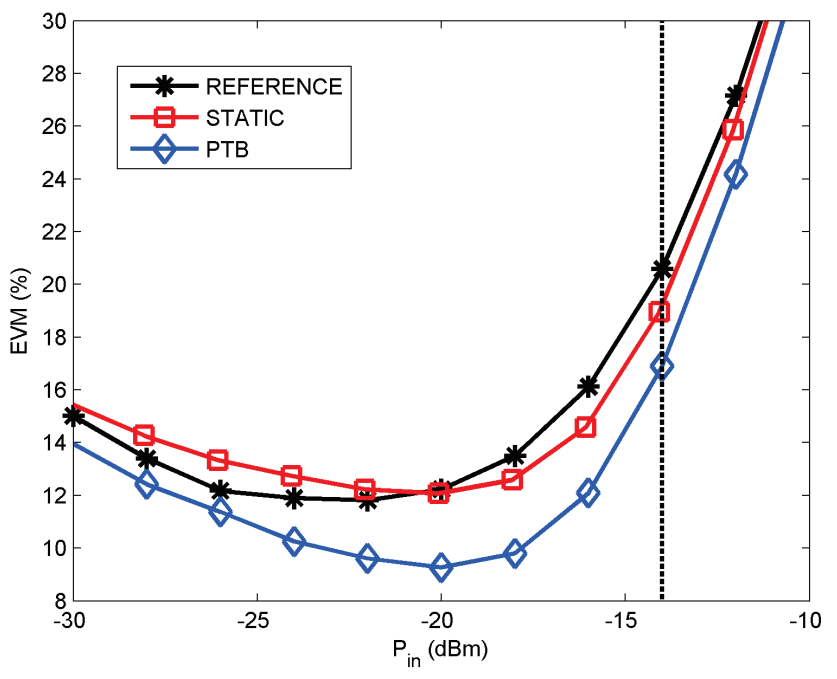

Fig. 2. Comparison of the performance of Static and PTB predistortion for an identification power of $\mathbf{P}_{\text {ref }}=-14 \mathrm{dBm}$.

In our previous study, we obtained the best performance/complexity compromise for $N_{3}=1$ and $M=5$ in the case of PTB predistortion [6]. In fact, beyond these values, there is no significant improvement in EVM. Fig. 2 shows the influence of the power injected in the amplifier on the performances of the Static and PTB predistorters. The results in Fig. 2 are relative to an identification at $P_{\text {ref }}=-14 \mathrm{dBm}$, close to the saturation regime. We now present validation results between $\mathrm{P}_{\text {in }}=-30 \mathrm{dBm}$ (low power) and $\mathrm{P}_{\text {in }}=-10 \mathrm{dBm}$ (high power). There is a significant margin of performance for the PTB structure, for comparable computational complexity. Overall, the PTB structure offers the best compromise, with the capability of lowering EVM over a wide range of transmitter parameters and low parametric complexity of predistorters.

\section{B. Joint Predistortion and PAPR Reduction}

In this communication we show that the combination of a predistorter with PAPR reduction (hard clipping) leads to better results than PAPR reduction alone. Hard clipping is 
straightforward to implement and optimize the algorithm relies on only one parameter - clipping ratio (CR) [9]. More elaborate soft clipping techniques, such as Wang companding [5], could also be considered but the authors believe this to be beyond the scope of this paper. Throughout the paper $\mathrm{CR}=5 \mathrm{~dB}$ is used. Fast grid-search shows this to be a good trade-off for all predistorters.

To illustrate the performance of the PAPR reduction and linearization blocks, separately or combined, we focus on the case of a QPSK/OFDM transmission. Fig. 3 shows the results obtained, in terms of EVM as a function of the input power $\left(\mathrm{P}_{\text {in }}\right)$ of the SOA, for various cases: conventional system (no PAPR reduction nor linearization), with PAPR reduction via clipping, with linearization via the Static or PTB and the combined scenarios. It can be clearly seen that a joint use of PAPR reduction and linearization offers significant performance gains. The joint use of the two approaches then achieves a very interesting compromise, enabling the range of operation of the transmitter to be significantly widened.

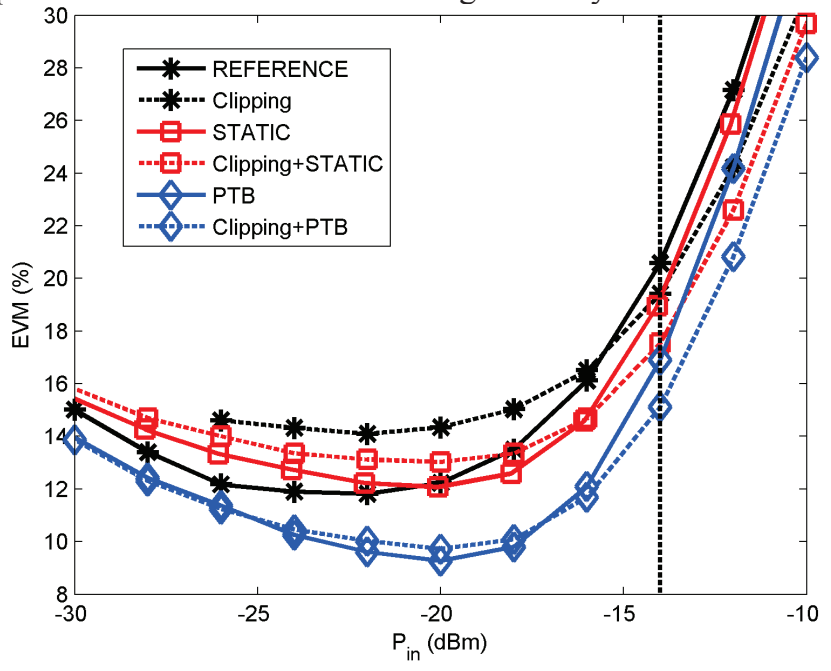

Fig. 3. EVM against input power at fixed $\mathrm{P}_{\text {ref }}$ at $-14 \mathrm{dBm}$.

\section{IQ Modulator Voltage Sweep}

Robustness against IQ modulator $V_{p p}$ is now studied for an identification at $V_{p p}=8 \mathrm{~V}$, with $P_{i n}=P_{r e f}=-14 \mathrm{dBm}$. Once determined, attenuation values are left unchanged. Robustness analysis will then consist in sweeping $V_{p p}$ from $1 \mathrm{~V}$ to $15 \mathrm{~V}$ and in assessing EVM. In Fig. 4.a, input power $P_{\text {in }}$ against $V_{p p}$ is plotted; a nonlinear dependence is observed. In Fig. 4.b, EVM against $V_{p p}$ is presented with $P_{\text {in }}=P_{\text {ref }}=-14$ $\mathrm{dBm}$. As expected, PTB performs better than simple static predistortion. We notice that EVM is high for $V_{p p}$ below $3 \mathrm{~V}$ due to low optical input power and a significant impact of amplified spontaneous emission noise on transmission quality. For $V_{p p}>8 \mathrm{~V}$ the $\mathrm{SOA}$ is operating close to its saturation point, EVM is then increasing due to nonlinear impairments. We clearly see that the joint predistortion and PAPR reduction offers a large gain in performance over a wide interval of $V_{p p}$, particularly when a PTB is used.

\section{Biasing Current Influence}

So far simulations were carried out for a fixed $150 \mathrm{~mA}$ injection current $\left(I_{\text {bias }}\right)$ setting. We now proceed to a biasing current sweep from $120 \mathrm{~mA}$ to $210 \mathrm{~mA}$, for $P_{\text {in }}=P_{\text {ref }}=$ $-14 \mathrm{dBm}$. In Fig. 5, we also observe that higher biasing current results in higher EVM values. PTB/clipping combination is once more providing the best performance; EVM at $220 \mathrm{~mA}$ is $24.89 \%$ for Static, $23.02 \%$ for PTB, $21.48 \%$ for joint Static and clipping and $19.80 \%$ for joint PTB and clipping.

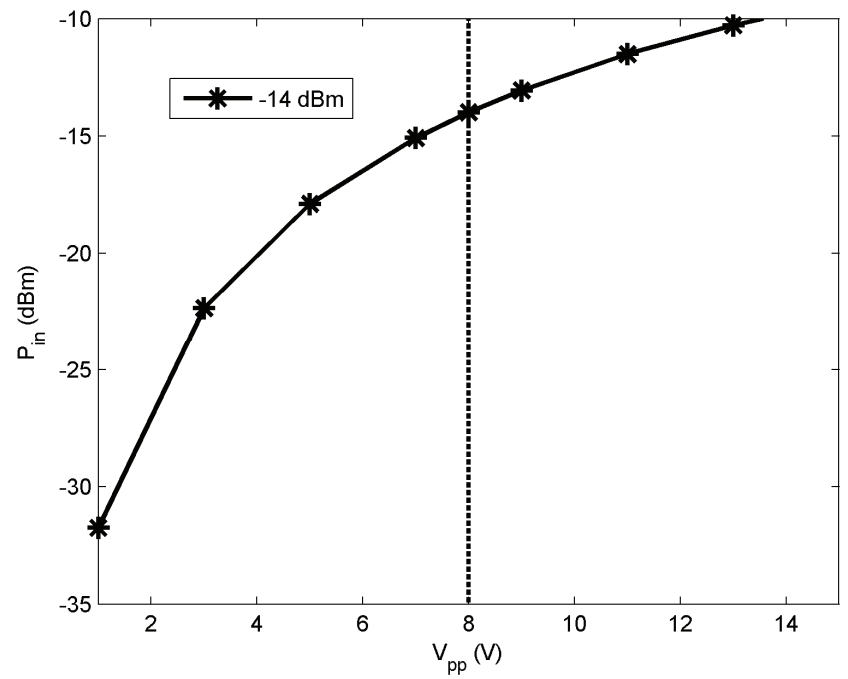

(a)

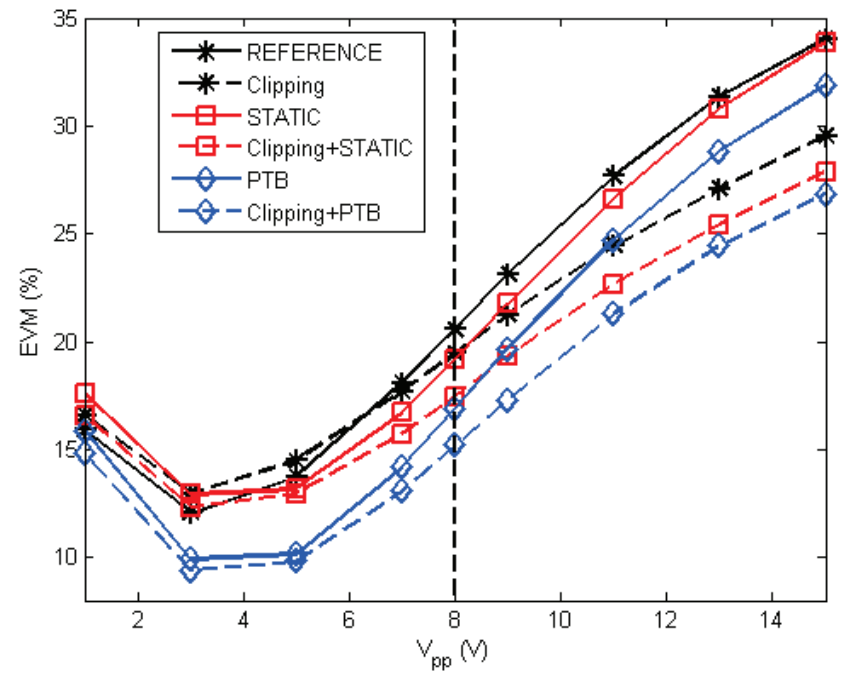

(b)

Fig. 4. (a) Input optical power against peak-to-peak voltage, (b) EVM against peak-to-peak voltage at fixed $\mathbf{P}_{\text {in }}$ to $-14 \mathrm{dBm}$.

\section{E. Wavelength Influence}

Previous simulations were carried out for a fixed laser wavelength $\lambda=1540 \mathrm{~nm}$. We can also study robustness with respect to $\lambda$ which is swept from 1500 to $1560 \mathrm{~nm}$. Once more, the combination of PTB and hard clipping offers the best performances (Fig 6). Note that the predistorter coefficients were identified at $\lambda=1540 \mathrm{~nm}$.

\section{F. Commutation and Multipoint Predistortions}

Besides the use of PAPR reduction the predistortion solutions discussed in [6] can be further improved by adopting strategies specifically aimed at increasing robustness. One such strategy is simultaneous identification at several operating 
points. This basically consists in performing the identification routine described in section II.C for concatenated input and output signals corresponding to different operating points. Least squares computation will intrinsically lead to an "average" predistorter exhibiting good performance for a wider range of parameters. The other strategy is even more straightforward. We suppose that various sets of coefficients are stored, for various values on input parameters (such as $P_{\text {in }}$ ), and a switching mechanism is implemented. This is not an unreasonable scenario in the context of an FPGA implementation. The results are collected in Fig. 7 and compared to the standard PTB predistorter. The multipoint predistorter was optimized at $P_{\text {in }}=P_{\text {ref } 1}=-26 \mathrm{dBm}$ and $P_{\text {in }}=P_{\text {ref } 2}=-14 \mathrm{dBm}$. It results in a very good compromise between robustness and performance over this power range (Fig. 7). The introduction of a switching mechanism with multiple coefficients sets further increases performance albeit at the cost of a slightly more complex implementation.

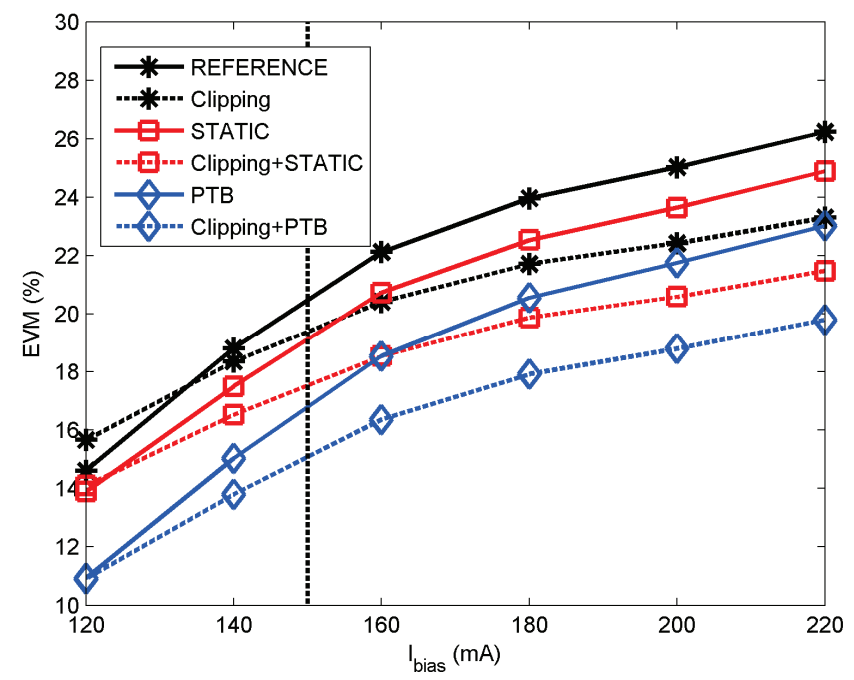

Fig. 5. EVM against bias current at fixed $\mathbf{P}_{\text {ref }}$ to $-14 \mathrm{dBm}$.

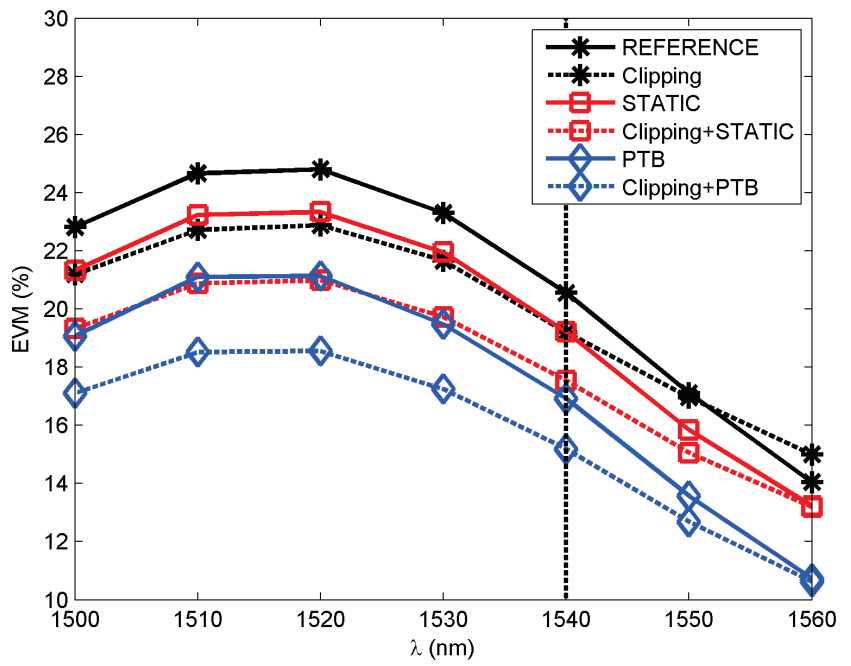

Fig. 6. EVM against input wavelength at fixed $\mathbf{P}_{\text {ref }}$ to $-14 \mathrm{dBm}$.

\section{CONCLUSION}

Various digital baseband predistorters have been investigated in this paper for improving the EVM performance of an SOA-based 17 Gbps CO-OFDM transmitter, while considering the criterion of robustness against system parametric variations. First of all, a simple memoryless (Static) predistorter was compared to a parallel structure (PTB) combining a static block and an MP predistorter. Then, it was shown that the combination of predistortion and PAPR reduction provided the better performance particularly when using PTB. This is an important result that completes the study previously published in [6]. Furthermore, the present paper introduced two very effective and simple strategies specifically aimed at increasing robustness.

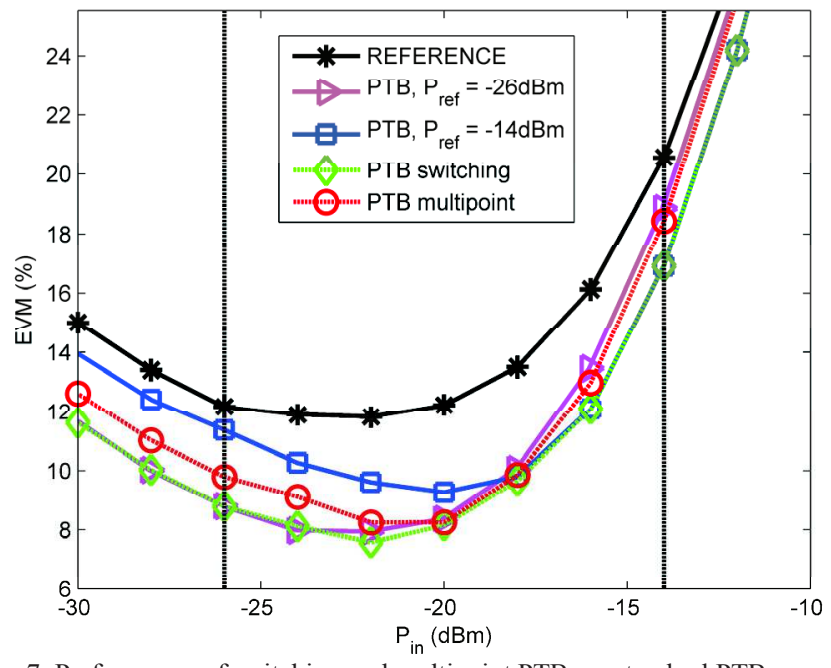

Fig. 7. Performance of switching and multipoint PTB vs. standard PTB.

\section{REFERENCES}

[1] N. Cvijetic, "OFDM for Next-Generation Optical Access Networks," IEEE J. Lightw. Technol., vol. 30, no. 4, pp. 384-398, February 2012.

[2] H. Khaleghi, P. Morel, A. Sharaiha, and T. Rampone, "Experimental Validation of Numerical Simulations and Performance Analysis of a Coherent Optical-OFDM Transmission System Employing a Semiconductor Optical Amplifier", IEEE J. Lightw. Technol., vol. 31, no. 1, pp. 161-170, January 2013.

[3] L. Guan and A. Zhu, "Green Communications: Digital Predistortion for Wideband RF Power Amplifiers", IEEE Microw. Mag., vol. 15, no. 7, pp. 84-99, November/December 2014.

[4] Z. Liu, M. Violas, and N. B. Carvalho, "Digital predistortion for RSOAs as external modulators in radio over fiber systems", Opt. Express, vol. 19, no. 18, pp. 17641-17646, 2011.

[5] S. Bejan, S. Azou, P. Morel, C. Diouf, M. Telescu, N. Tanguy, and A. Sharaiha, "A joint Linearization/Companding Approach for Improving a CO-OFDM Transmitter", IEEE Photon. Technol. Lett., vol. 27, no. 20, pp. 2162-2165, 2015.

[6] C. Diouf, M. Younes, A. Noaja, S. Azou, M. Telescu, P. Morel, and N. Tanguy, "Robustness analysis of a parallel two-box digital polynomial predistorter for an SOA-based CO-OFDM system", Opt. Commun., vol. 402, pp. 442-452, nov 2017.

[7] E. Agrell, et al., "Roadmap of optical communications", J. Opt., vol. 18, no. 6, 063002, 2016.

[8] C. C. Paige and M. A. Saunders, "LSQR: An algorithm for sparse linear equations and sparse least squares", ACM Trans. Math. Softw., vol. 8 , no. 1, pp. 43-71, 1982.

[9] D. Kim and G. L. Stuber, "Clipping Noise Mitigation for OFDM by Decision-Aided Reconstruction”, IEEE Commun. Lett., vol. 3, no. 1, pp. 4-6, Jan. 1999. 